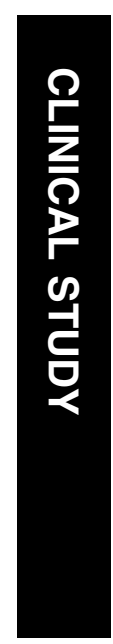

\title{
Reduction of pegaptanib loss during intravitreal delivery using an oblique injection technique
}

${ }^{1}$ Department of Ophthalmology, Hospital 'Príncipe de Asturias', Alcalá de Henares, Madrid, Spain

${ }^{2}$ Department of Ophthalmology, Hospital 'La Mancha Centro', Alcazar de San Juan, Ciudad Real, Spain

Correspondence:

L López-Guajardo,

Department of

Ophthalmology,

Hospital 'Príncipe de

Asturias',

Avda. Crra. Alcala-Meco s/n

Alcalá de Henares,

Madrid

28805,

Spain

Tel: + 34-677-49-17-26;

Fax: +91-554-14-80.

E-mail: Ilguajardo@

icam.es

Received: 17 April 2007

Accepted in revised form:

5 November 2007

Published online: 18 January 2008

Neither of the authors has a proprietary or financial interest in any material or method mentioned. No funding was received.

\begin{abstract}
Purpose To develop an injection technique that reduces drug loss occurring due to reflux and consequent subconjunctival bleb formation after standard intravitreal pegaptanib injection.

Methods Prospective interventional case series report. The 27-gauge needle is inserted obliquely via pars plana to create a valved wound. The rate of subconjunctival bleb formation is evaluated performing ultrasound biomicroscopy, $\frac{1}{2} \mathrm{~h}$ after intravitreal drug delivery comparing standard (straight) with this new described injection technique (oblique) using Fisher's exact test.

Results Eyes on which oblique injection technique was performed developed significantly less subconjunctival bleb than those treated with the standard technique. The rate of injection-related complications (retinal detachment, vitreous haemorrhage, traumatic cataract, and endophthalmitis) in our small series was similar between both groups. Conclusions The use of this technique can result in reduced drug loss after intravitreal pegaptanib injection. Larger studies are needed to determine if the rate of complications associated with intravitreal injections, especially endophthalmitis, is lowered with this technique.
\end{abstract}

Eye (2008) 22, 430-433; doi:10.1038/sj.eye.6703054; published online 18 January 2008

Keywords: intravitreal injection; oblique injection; pegaptanib; subconjunctival bleb

\section{Introduction}

Intravitreal drug delivery is the usual method for anti-VEGF (vascular endothelial growth
L Lopez-Guajardo ${ }^{1}$, FG del Valle², JP Moreno ${ }^{1}$ and MA Teus ${ }^{1}$ factor) therapy. The first approved drug, pegaptanib sodium (Pfizer Inc., New York, NY, USA), comes in a precharged syringe (BD Hypak; Becton Dickinson, Franklin Lakes, NJ, USA) with a 27 gauge $\times \frac{1}{2}$ inch beveled needle. The standard intravitreal injection technique (perpendicular to scleral surface-direct technique), ${ }^{1}$ in many cases results in reflux of liquified vitreous which will surely be mixed with some quantity of drug. The small volume administered $(0.09 \mathrm{ml})$ may result in a significant part of the dose being lost due to reflux and consequent subconjunctival bleb formation.

In an experimental pig eye model, we injected $0.09 \mathrm{ml}$ of methylene blue, and reflux of the injected liquid substance was demonstrated by staining of the subconjunctival bleb (Figure 1). We can assume that this can also be the case with pegaptanib in clinical situations.

A tunnelled biplanar injection has already been described for intravitreal avastin and triamcinolone delivery. ${ }^{2}$ Oblique incisions have already been described ${ }^{3}$ in microincisional pars plana vitrectomy.

\section{Materials and methods}

We performed a prospective interventional series in which consecutive patients scheduled for intravitreal pegaptanib injection were randomized to receive injections performed either with the standard (direct) or oblique technique (see below). Informed consent was signed by all patients.

The injection technique was performed following accepted guidelines. ${ }^{1,4}$ For the oblique injection technique, (Figure 2) the eye was secured with a pair of forceps grasping conjunctiva next to the limbus to avoid rotation when the needle was being inserted. The needle 


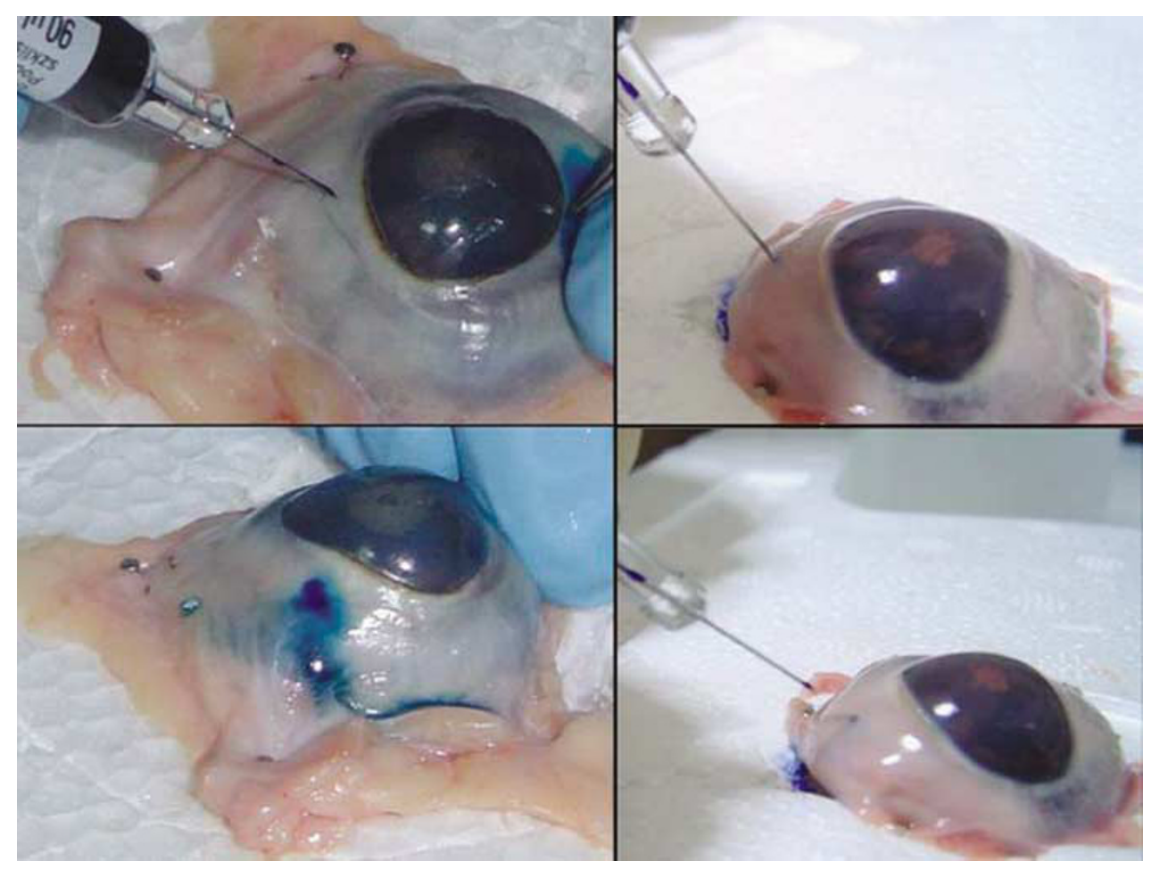

Figure 1 Top left: pig eye before injection perpendicular to scleral surface of methylene blue ( $0.09 \mathrm{ml})$ using same syringe (BD Hypak) with 27-gauge needle as pegaptanib. Bottom left: subconjunctival blue bleb with some extraocular dye accumulated over conjunctival folds as a result of reflux of part of the intravitreally injected methylene blue. Top right: pig eye before performing oblique intraocular methylene blue injection using BD Hypak. Bottom right: minimal subconjunctival blue bleb formation after intraocular dye (0.09 ml) injection.

was then inserted bevel-up, penetrating the globe obliquely (angle $40-30^{\circ}$ with scleral plane) and at the same time pointing to the six o'clock equatorial area (to avoid contact with proximal peripheral retina or damage to the lens - phakic eyes) up to approximately half needle length. This creates a valved incision at the scleral level. Pegaptanib was then injected and after the forceps was positioned over the created wound and counterpressure was exerted at the injection site to achieve immediate closure at the moment of needle withdrawal, as described by Shimada ${ }^{5}$ for 25 -gauge vitrectomy oblique sclerotomy. Paracentesis was not performed to reduce intraocular pressure.

Half an hour after the injection, ultrasound biomicroscopy (UBM) was performed to evaluate the presence of subconjunctival bleb (Figure 3), which was defined as the separation of conjunctiva from superficial sclera by an echographically empty space wider than one-third of scleral thickness. If a subconjunctival bleb was caused by post-injection hyposphagma (confirmed by direct visualization), it was not considered as the bleb was not caused by intraocular reflux. After performing UBM, the eye was patched for $2 \mathrm{~h}$.

The biomicroscopic image sequences obtained were reviewed and one snapshot was selected for each eye. The snapshots were then presented in a masked fashion to one of the authors who determined the presence/ absence of subconjunctival bleb.

Injection-related complications ${ }^{6}$ were recorded at least for the next 6 weeks.

We certify that all applicable institutional and governmental regulations concerning the ethical use of human volunteers and animals were followed during this research.

\section{Results}

We performed direct injection technique in 22 eyes and oblique injection technique in 25 eyes. A bleb was identified in 15 of the direct $(68 \%)$ and 9 of the oblique group eyes $(36 \%)$, a difference that was statistically significant ( $P=0.041$; Fisher's exact test).

No post-injection retinal detachment, endophthalmitis, traumatic cataract, or vitreous haemorrhage were recorded in the standard or oblique technique groups.

\section{Discussion}

Valved scleral self-sealing incisions, ${ }^{7,8}$ as well as valved corneal incisions, ${ }^{9}$ are favoured in their closing mechanism by intraocular pressure. After performing an 


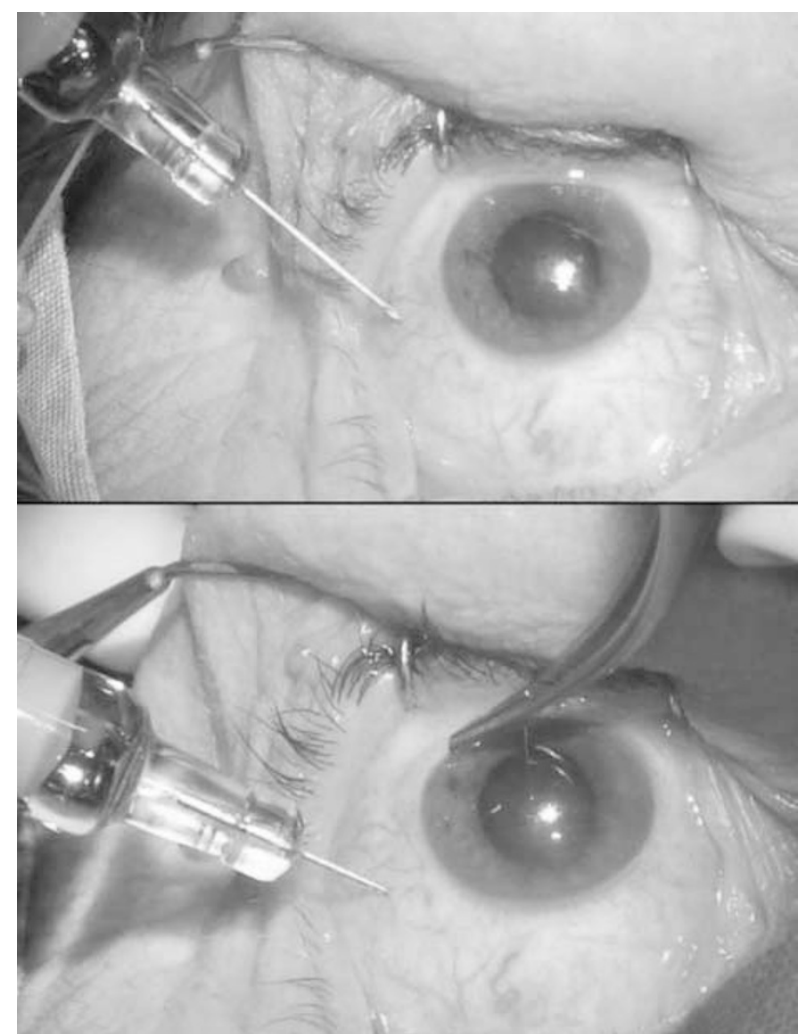

Figure 2 Top: before oblique injection of pegaptanib with needle bevel-up, oriented tangentially to globe (angle $40-30^{\circ}$ ), and pointing to 'six' o clock equatorial area. Bottom: eye secured from rotating by forceps holding conjunctiva close to the limbus, and needle inserted approximately half length.

intraocular injection, intraocular pressure is usually elevated, and we believe that the same mechanism that helps close valved incisions occurs while performing an oblique pars plana injection. If the direct injection technique is used (that is, perpendicular to the scleral surface), this same increased pressure does not aid in closing the wound but favours the opening and intraocular exit of contents to equilibrate the pressure gradient.

From our results, we could conclude that the oblique injection technique may reduce pegaptanib loss during intraocular administration, which may also aid in better response to treatment. The number of patients studied does not reveal significant differences with regard to complications related to this type of intraocular administration, but we believe that larger studies are needed to determine if there is any advantage in performing pegaptanib injections with the oblique technique with regard to injection-related complications and treatment efficacy as possibly less drug is lost due to reflux.

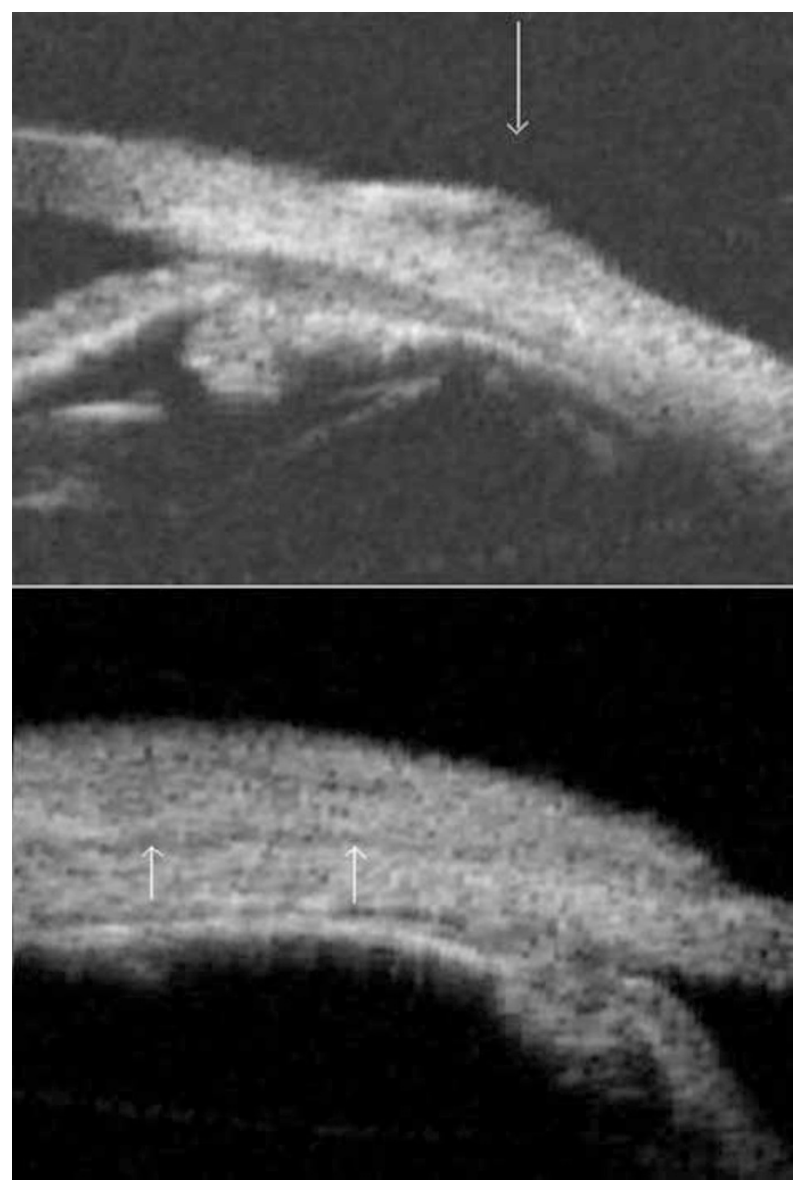

Figure 3 Top: ultrasound biomicroscopic image of subconjunctival bleb. Arrow points at ecographically empty space between conjunctiva and superficial sclera located at injection site. Bottom: ecographically dense subconjunctival bleb (arrows indicate superficial scleral level). This bleb is formed by blood coming from epiescleral/conjunctival vessels injured during needle insertion. As the contents of this bleb are not of intraocular origin, this bleb is not considered for the purpose of this study.

\section{References}

1 Aiello LP, Brucker AJ, Chang S, Cunningham ET, D'amico DJ, Flynn HW et al. Evolving guidelines for intravitreous injections. Retina 2004; 24: S3-S19.

2 Rodrigues EB, Meyer CH, Grumann A, Shiroma H, Aguni JS, Farah ME. Tunnelled scleral incision to prevent vitreal reflux after intravitreal injection. Am J Ophthalmol 2007; 143: 1035-1037.

3 Lopez-Guajardo L, Pareja-Esteban J, Teus-Guezala MA. Oblique sclerotomy technique for prevention of incompetent wound closure in transconjunctival 25-gauge vitrectomy. Am J Ophthalmol 2006; 141: 1154-1156.

4 Ta N. Minimizing the risk of endophthalmitis following intravitreous injections. Retina 2004; 24: 699-705.

5 Shimada H, Nakashizuka H, Mori R, Mizutani Y, Hattori T. 25-gauge scleral tunnel transconjunctival vitrectomy. Am J Ophthalmol 2006; 142: 871-873. 
6 Jager RD, Aiello LP, Patel SC, Cunningham ET. Risks of intravitreous injection: a comprehensive review. Retina 2004, 24: 676-698.

7 Yeshurun I, Rock T, Bartov E. Modified sutureless sclerotomies for pars plana vitrectomy. Am J Ophthalmol 2004; 138: $866-867$.
8 Chen JC. Sutureless pars plana vitrectomy through self-sealing sclerotomies. Arch Ophthalmol 1996; 114: 1273-1275.

9 Ernest PH, Kiessling LA, Lavery KT. Relative strength of cataract incisions in cadaver eyes. J Cataract Refract Surg 1991; 17(Suppl): 668-671. 